\title{
Compound Parabolic Concentrator Design: Assessment of Performance Enhancement for Building-Integrated Photovoltaic Systems
}

\author{
Sander Saers, Anita Ortega*, Subhash Chandra, Sarah McCormack \\ Solar Energy Applications Group, Department of Civil, Structural and Environmental Engineering, Trinity \\ College Dublin, IRELAND \\ Email:ortegaa@tcd.ie
}

\begin{abstract}
Improvement of state-of-the-art sustainable energy technology is required to accelerate the essential global transition away from fossil fuel energy sources. One potential method to increase the energy output of building integrated photovoltaics (BIPV) is achieved by using parabolic reflectors, commonly known as compound parabolic concentrators (CPC). These curved mirrors allow incoming sunlight to be focused onto adjacent solar panels, thereby increasing irradiance. Although the concentrating effects of CPCs have been demonstrated in multiple studies, large scale adoption of BIPV-CPC systems has been held back due to variability in performance and added financial cost. This study aims to assess the technical performance of $B I P V-C P C$ systems through comparison of varying designs characteristics and environmental conditions using simulation models for two locations Dublin, Ireland and Ferrara, Italy.
\end{abstract}

KEYWORDS: Photovoltaics, Compound parabolic concentrator, Building Integration

\section{INTRODUCTION}

Photovoltaic (PV) technology allows solar radiation to be converted to electricity, providing means to utilise this near-boundless energy source. Due to continuously improving production cost and financial incentives, PV installations have become increasingly common in domestic and public buildings. Building integrated PV (BIPV) is considered an effective strategy for increasing PV system efficiency, as the power generated is in close proximity to the user and no additional land area is required. However, most silicon PV panel systems still only achieve an energy conversion efficiency between $17-22 \%$.

Compound parabolic concentrator (CPC) technology can further increase the energy output of solar panels by concentrating solar radiation onto the absorber surface. CPCs contain curved reflectors which act like a solar funnel, focusing sunlight from a larger inlet surface onto the PV panel. Previous studies have shown the benefits of the application of CPC technology, however it has not yet been adopted on a large scale.

The aim of this study is to assess the technical performance enhancement of the application of BIPV-CPC systems and to create and apply simulation models to estimate the technical performance of the selected CPC types and locations and to determine the economic benefit of the chosen CPCs in electricity savings and feed in tariffs (FIT) in each location; e) to present and discuss the simulation model outcomes regarding technical and economic performance.

\section{METHODOLOGY}

Table 1 shows an overview of the reviewed building BIPV-CPC studies (adapted from [1]), and was expanded to include recent studies. It includes the location and publication date of the study, design characteristics regarding symmetry, angle $(\theta)$ and the concentration ratio. Most important design parameters are (i) Acceptance angle, concentration ratio and truncation The acceptance angles ( $\theta)$ of the CPC dictate the technical performance of the design. A wide $\theta$ will result is a low geometrical concentration ratio. Conversely, a narrow $\theta$ will result in a high geometrical concentration ratio. Additionally, the CPC can be truncated to reduce the installation size of the system. The truncation length will however also influence the performance. The 
location and orientation should be taken into consideration during the determination of truncation length, and $\theta$ should be based on location and orientation [2].

Table 1: Overview of BICPC literature (adapted from Tian et al., 2018)

\begin{tabular}{|l|l|l|l|l|l|}
\hline Year & \multicolumn{1}{|c|}{$\begin{array}{c}\text { CPC } \\
\text { design }\end{array}$} & \multicolumn{1}{|c|}{$\begin{array}{c}\text { Study } \\
\text { type }\end{array}$} & $\begin{array}{c}\text { Conc. } \\
\text { Ratio }\end{array}$ & $\begin{array}{l}\text { Half- } \\
\text { accept. } \\
\text { Angle } \\
(\boldsymbol{\theta})\end{array}$ & Ref \\
\hline $\mathbf{2 0 0 0}$ & $\begin{array}{l}\text { Dielectric filled } \\
\text { symmetrical }\end{array}$ & $\begin{array}{l}\text { Conceptual } \\
\text { design }\end{array}$ & 2.46 & 37 & {$[3]$} \\
\hline $\mathbf{2 0 0 0}$ & $\begin{array}{l}\text { Dielectric filled } \\
\text { asymmetrical }\end{array}$ & $\begin{array}{l}\text { Conceptual } \\
\text { design }\end{array}$ & 2.96 & 36.4 & {$[3]$} \\
\hline $\mathbf{2 0 0 4}$ & Asymmetrical & $\begin{array}{l}\text { Prototype } \\
\text { test outdoor }\end{array}$ & 2.01 & $50-0$ & {$[4]$} \\
\hline $\mathbf{2 0 0 7}$ & Asymmetrical & $\begin{array}{l}\text { Prototype } \\
\text { test outdoor }\end{array}$ & 2.45 & $37-0$ & {$[5]$} \\
\hline $\mathbf{2 0 1 4}$ & $\begin{array}{l}\text { Dielectric filled } \\
\text { symmetrical }\end{array}$ & $\begin{array}{l}\text { Prototype } \\
\text { test }\end{array}$ & 2.8 & 55 & {$[6]$} \\
\hline $\mathbf{2 0 1 4}$ & $\begin{array}{l}\text { Dielectric filled } \\
\text { asymmetrical }\end{array}$ & $\begin{array}{l}\text { Prototype } \\
\text { test }\end{array}$ & 2.8 & $55-0$ & {$[7]$} \\
\hline $\mathbf{2 0 1 5}$ & $\begin{array}{l}\text { Dielectric filled } \\
\text { asymmetrical }\end{array}$ & $\begin{array}{l}\text { Prototype } \\
\text { test outdoor }\end{array}$ & 2.8 & $55-0$ & {$[7]$} \\
\hline $\mathbf{2 0 1 7}$ & Asymmetrical & $\begin{array}{l}\text { Prototype } \\
\text { test outdoor }\end{array}$ & 1.83 & $60-0$ & {$[8]$} \\
\hline
\end{tabular}

(ii) Location and orientation: Location and orientation of the system globally as well as locally must be included in the process of design decisions. As the research will be performed in Dublin Ireland, the Irish solar climate conditions such as the ratio of direct and diffuse solar radiation, and the local solar trajectories throughout the year, will dictate these design choices. The desired and available azimuth and the tilt angles for the PV-CPC system are also influencing factors. (iii) Temperature and uniformity of radiative flux distribution: To optimise the efficiency of the CPC, several physical and electrical influences should be considered as well: An increase in temperature will reduce the efficiency of a silicon PV cell. Therefore, the temperature gain should be mitigated. This can be controlled passively through designed cooling ribs or actively with actuated fans. On top of the general negative effect of heating on PV cell efficiency, the uniformity of the distribution of the radiative flux onto the cells also has a significant impact on the efficiency. Because the cells are arranged in series, the cell producing the lowest amount of power will determine the power produced for the entire panel/array. Therefore, an even distribution of radiation is required for optimal performance. (iv) Materials and optical gaps: The optical efficiency of the reflective/refractive materials used for the concentrator should be as high as possible for optimal performance. This increases the amount of radiation focused onto the absorber and reduces heat gain. Furthermore, to channel the maximum amount of solar radiation towards the PV surface, the solar radiation 'leakage' through optical gaps in the design should be minimised, for instance through the use of dielectric film.

\section{MODEL DEVELOPMENT}

A simulation approach has been chosen to find the optimal CPC shape and orientation for enhancement of solar radiation onto a photovoltaic surface. To achieve this, separate simulation models were created. In these models, multiple CPC variations were analysed in varying virtual circumstances. The CPC design variations were assessed in simulation conditions representing two cities with different climates. Both direct solar radiation and diffuse (indirect) solar radiation were analysed. Table 2, presents a complete overview of all 22 CPC variations included in the simulations. 


\begin{tabular}{|c|c|c|c|c|}
\hline \multicolumn{5}{|c|}{ CPC VARIATIONS } \\
\hline Reference code & $\begin{array}{c}\text { Acceptance } \\
\text { angle } \theta\end{array}$ & $\begin{array}{c}\text { Panel } \\
\text { orientation }\end{array}$ & $\begin{array}{l}\text { Visual concept } \\
\text { representation }\end{array}$ & $\begin{array}{l}\text { CPC axes } \\
\text { symmetry }\end{array}$ \\
\hline S-H-80 & $80^{\circ}$ & \multirow{4}{*}{ Horizontal } & & \multirow{12}{*}{ Symmetrical } \\
\hline S-H-100 & $100^{\circ}$ & & & \\
\hline S-H-120 & $120^{\circ}$ & & & \\
\hline S-H-140 & $140^{\circ}$ & & & \\
\hline S-V-80 & $80^{\circ}$ & \multirow{4}{*}{ Vertical } & & \\
\hline S-V-100 & $100^{\circ}$ & & & \\
\hline S-V-120 & $120^{\circ}$ & & & \\
\hline S-V-140 & $140^{\circ}$ & & & \\
\hline S-T- 80 & $80^{\circ}$ & \multirow{4}{*}{$\begin{array}{c}\text { Tilted } \\
\text { (fixed optimal } \\
\text { sun facing angle) }\end{array}$} & & \\
\hline S-T-100 & $100^{\circ}$ & & & \\
\hline S-T-120 & $120^{\circ}$ & & & \\
\hline S-T-140 & $140^{\circ}$ & & ] & \\
\hline AS-H-40 & $40^{\circ}+19^{\circ}$ & \multirow{4}{*}{ Horizontal } & & \multirow{8}{*}{ Asymmetrical } \\
\hline AS-H-50 & $50^{\circ}+32^{\circ}$ & & & \\
\hline AS-H-60 & $60^{\circ}+48^{\circ}$ & & & \\
\hline AS-H-70 & $70^{\circ}+64^{\circ}$ & & & \\
\hline AS-V-40 & $40^{\circ}+19^{\circ}$ & \multirow{4}{*}{ Vertical } & & \\
\hline AS-V-50 & $50^{\circ}+32^{\circ}$ & & & \\
\hline AS-V-60 & $60^{\circ}+48^{\circ}$ & & & \\
\hline AS-V-70 & $70^{\circ}+64^{\circ}$ & & & \\
\hline
\end{tabular}

Table 2: CPC variations for ray-tracing simulation

Two locations were selected for assessment: Dublin (Ireland) and Ferrara (Italy). 3D models for each CPC type were created for the ray-tracing simulation. Solidworks was used as a CAD modelling software to generate these models. Using the software, a 2D profile sketch of each CPC was drawn and subsequently extruded $500 \mathrm{~mm}$ into a 3D shape. This 3D model was then imported into a ray-tracing software to perform the ray-tracing analyses. TracePro, a software by Lambda Research Corporation, was utilised to perform the ray-tracing analyses for diffuse and direct solar irradiation onto the 3D model.

Two locations were selected for assessment: Dublin (Ireland) and Ferrara (Italy). 3D models for each CPC type were created for the ray-tracing simulation. Solidworks was used as a CAD modelling software to generate these models. Using the software, a 2D profile sketch of each CPC was drawn and subsequently extruded $500 \mathrm{~mm}$ into a 3D shape. This 3D model was then imported into a ray-tracing software to perform the ray-tracing analyses. TracePro, a software by Lambda Research Corporation, was utilised to perform the ray-tracing analyses for diffuse and direct solar irradiation onto the 3D model.

The parabolic reflector surfaces bordering each side of the PV panel are attributed a $95 \%$ reflection and $5 \%$ absorption, PV panel surface was treated as a perfect absorber exit surface. Although this is not realistic for the purpose of simulation of PV panel output, it will allow adequate assessment of the effectiveness of the CPC performance regardless of PV panel quality and efficiency. The inner side panels is attributed perfect mirror material property (100\% reflection) to prevent loss of irradiation through the sides: Optimal panel tilt angle $(\alpha)$ per location was calculated using the NASA database (NASA, 2020), which includes the monthly optimal panel tilt angle. The optimal fixed $\alpha$ for the entire year ( $\left.\alpha_{\text {opt.year }}\right)$ was found through the sum of the product of the monthly optimal angle ( $\alpha_{\text {opt.month }}$ ) and the corresponding irradiation (Iopt.month), consecutively divided by the sum of I opt.month. CPC 
performance was simulated in conditions resembling three times of the year: (i) Summer solstice; (ii) Spring/Autumn equinox; (iii) Winter solstice. From these three points in the year an extrapolation for an estimated average annual performance was made.

Results from the estimated diffuse and direct irradiance derived from the simulation model were combined and the cumulative irradiance (Itotal) is estimated per location. The results are shown in Tables 2 \&3 where seasonal and annual average $I_{\text {direct }}$, Idirect and $I_{\text {total }}$ are presented along with concentration ratio.

For all of the CPC designs assessed in this study, the absorber surface is $125 \times 500 \mathrm{~mm}$ and the inlet surfaces are larger than this. Therefore, the geometrical concentration ratio is always positive $(>100 \%)$. The inlet surface, and herewith the geometrical concentration ratio, is increased with decreasing acceptance angles $(\theta)$ in the CPC design. Amongst the CPC designs that were assessed, this ratio varies between $133 \%$ and $338 \%$.

\begin{tabular}{|c|c|c|c|c|c|c|c|c|c|c|c|c|c|c|c|c|c|}
\hline & \multirow{2}{*}{\multicolumn{2}{|c|}{$\begin{array}{c}\text { Symmetrical } \\
\text { Tilted } 40^{\circ}\end{array}$}} & \multicolumn{3}{|c|}{ Rer-T40 } & \multicolumn{3}{|c|}{ S-Ts0-000 } & \multicolumn{3}{|c|}{ 5.740. 150} & \multicolumn{3}{|c|}{$2-T=0-1200$} & \multicolumn{3}{|c|}{$3 T 40-140$} \\
\hline & & & wNrn & roNe & sasur & wNm & rover & sums & worts & $\cos \alpha$ & sMur & wsm & ronos & smens & wNmR & $\tan x$ & suma \\
\hline \multirow{6}{*}{ 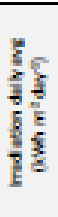 } & \multirow{2}{*}{ Direst } & Semon & $0 . \omega$ & 1.08 & 200 & 0.57 & sea & 200 & 0.00 & 100 & 212 & נתם & $1 x$ & 2.15 & נתם & 200 & 2.11 \\
\hline & & Year & & 1.43 & & & $2 x$ & (19es) & & 265 & (דתוב) & & 2.38 & $(157 \times)$ & & 200 & (1)rs) \\
\hline & \multirow{2}{*}{ Dithas } & Semon & $0.2 n$ & 1.11 & 200 & $0.2 n$ & 1.11 & 200 & $0.2 \mathrm{an}$ & 1.11 & 200 & ora & 1.11 & 200 & 0.20 & 111 & 200 \\
\hline & & Yesr & & 1.15 & & & 1.15 & & & 1.15 & & & 1.15 & & & 1.15 & \\
\hline & \multirow{2}{*}{ Towal } & Semon & o.s. & $2 \pi$ & 4.17 & a.ss & 2.75 & 4.10 & 0.96 & 5.02 & 421 & 101 & 430 & 4.25 & 101 & $2 \pi$ & 422 \\
\hline & & Yeser & & $2 \mathrm{CH}$ & & & 4.11 & (180) & & 100 & (340) & & 290 & $(112 \times)$ & & 220 & (1218) \\
\hline
\end{tabular}

\begin{tabular}{|c|c|c|c|c|c|c|c|c|c|c|c|c|c|c|c|c|c|}
\hline & $\mathrm{Sym}$ & trical & & nerr.y & & & $-2-100$ & & & $5 \%-100$ & & & $3-4.120$ & & & $5-k-100$ & \\
\hline & & & wNTn & $\operatorname{tos} \alpha$ & SNove & WNT & rONA & SUuR & Worts & $\cos \alpha$ & SMUR & wsth & moner & smous & WNTR & tons & suma \\
\hline \multirow{6}{*}{ 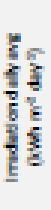 } & \multirow{2}{*}{ Dirst } & Stwon & 0.70 & 1.12 & 2.15 & 1.24 & 0.51 & 0.11 & 1.07 & 0.00 & 0.11 & 0.97 & 1.16 & 0.11 & and & 128 & Q.4n \\
\hline & & Year & & 1.12 & & & 0.57 & (515) & & 0.76 & (ets) & & 0.8 & $(70 x)$ & & a.se & $(\operatorname{arn} x)$ \\
\hline & \multirow{2}{*}{ dithes } & Stren & a.s & 0.72 & 10 & a.dn & 0.72 & 1.98 & 0.19 & 0.72 & 1.38 & 0.19 & $a r$ & 1.95 & a.d & $a r$ & 1.25 \\
\hline & & Year & & 0.74 & & & 0.74 & & & 0.74 & & & 0.74 & & & 0.74 & \\
\hline & \multirow{2}{*}{ Total } & Samon & ans & 2.00 & 2.0 & 1.12 & 1.23 & 2.46 & 1.78 & 108 & $2 \mathrm{LCC}$ & 1.15 & $1 \mathrm{men}$ & 1.16 & $10 x$ & 201 & 1.92 \\
\hline & & Tear & & 1.06 & & & 1.11 & $(7 \infty)$ & & 1.20 & (n1s) & & 1.50 & $(\mathrm{nrx})$ & & $1 \pi$ & (205) \\
\hline
\end{tabular}

\begin{tabular}{|c|c|c|c|c|c|c|c|c|c|c|c|c|c|c|c|c|c|}
\hline & Sym & trical & & Err-H & & & $311-\infty$ & & & $=1100$ & & & $=11.120$ & & & $5-11-260$ & \\
\hline & Ho & atal & whrn & roser. & sмMr & พNTR & $\operatorname{ros} x$ & suun & WNTR & $\cos \alpha$ & SMUR & wish & ponot & sever & WNTR & $\operatorname{ton} \alpha$ & suma \\
\hline \multirow{6}{*}{ 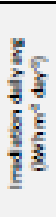 } & \multirow{2}{*}{ Dirsa } & Servon & 0.28 & a.s & 1.77 & 0.04 & a.an & 19 & 0.00 & a.ta & 200 & $O D 1$ & 000 & 200 & 0.01 & $\alpha a$ & 201 \\
\hline & & Yest & & 0.92 & & & 0.54 & (20) & & 0.50 & (Bes) & & מ.ת & $(m x)$ & & 0.00 & $(90 \mathrm{CS})$ \\
\hline & \multirow{2}{*}{ Difusen } & Sexton & $a x$ & 1.40 & 200 & $a x$ & 1.40 & 200 & 0.96 & 100 & 200 & 0.36 & 100 & 200 & $a x$ & 100 & 203 \\
\hline & & Yest & & 1.44 & & & 1.44 & & & 160 & & & 140 & & & 140 & \\
\hline & \multirow{2}{*}{ Total } & Sewon & 0.52 & 2.11 & 4.46 & 0.57 & 1.61 & 450 & 0.97 & 1.61 & 471 & 0.97 & 1.0 & 4.75 & 0.37 & 200 & 470 \\
\hline & & Yesr & & 2.40 & & & 202 & (DN) & & 207 & (ne) & & 271 & $(25 X)$ & & 231 & $(9 \infty \times)$ \\
\hline
\end{tabular}

\begin{tabular}{|c|c|c|c|c|c|c|c|c|c|c|c|c|c|c|c|c|c|}
\hline & \multirow{2}{*}{\multicolumn{2}{|c|}{$\begin{array}{c}\text { Asymmetrical } \\
\text { Vertical }\end{array}$}} & \multicolumn{3}{|c|}{ ner.y } & \multicolumn{3}{|c|}{$n \in V+0$} & \multicolumn{3}{|c|}{$N-v-90$} & \multicolumn{3}{|c|}{$N-\psi \cdot \omega$} & \multicolumn{3}{|c|}{ nev-so } \\
\hline & & & wNrn & tons & sмvis & พพm & roser & sMun & worn & soso & sMur & with & noner & save & WNTR & roner & suma \\
\hline \multirow{6}{*}{ 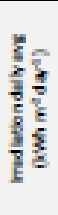 } & \multirow{2}{*}{ Dirst } & Semon & 0.70 & 1.12 & 2.15 & 1.67 & 2.20 & 0.11 & 2.4 & 2.15 & 0.00 & 1.29 & 211 & 0.0 & 101 & 191 & 100 \\
\hline & & Yesr & & 1.12 & & & 1.53 & (130) & & 100 & (245) & & 158 & $(120 \times 5)$ & & 1.40 & (1208) \\
\hline & \multirow{2}{*}{ Difrus } & Semon & 0.18 & 0.72 & 1.85 & a.19 & 0.72 & $1 . x$ & 0.19 & 0.72 & 1.98 & 0.19 & $0 \pi$ & 1.95 & 0.18 & $a r$ & 1.98 \\
\hline & & Year & & 0.74 & & & 0.74 & & & 0.74 & & & 0.74 & & & 0.74 & \\
\hline & \multirow{2}{*}{ Toral } & Semon & o.sn & 200 & 200 & 1.15 & 2.07 & 1.46 & 1.06 & 2.97 & 1.07 & 2.41 & 200 & 200 & 1.10 & 202 & 2.4 \\
\hline & & Fear & & $1 . .6$ & & & 2.26 & (128x) & & 2.34 & (120) & & 229 & $(122 x)$ & & 222 & (HEN) \\
\hline
\end{tabular}

\begin{tabular}{|c|c|c|c|c|c|c|c|c|c|c|c|c|c|c|c|c|c|}
\hline & Asym & trical & & Err-H & & & $N-H 1-40$ & & & N-11-50 & & & NSHED & & & $M E+11-70$ & \\
\hline & & atal & whrn & tose & sмsur & พผm & toNK & suun & WNTn & $\cos \alpha$ & SMUR & wims & Doner & sмM & WNTR & rover & suma \\
\hline \multirow{6}{*}{ 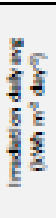 } & \multirow{2}{*}{ Dirst } & Strion & 0.28 & and & 1.77 & 0.04 & 0.20 & 1.95 & 0.01 & 100 & 217 & 0.01 & 100 & 234 & 0.01 & 122 & 2.46 \\
\hline & & Yesr & & 0.12 & & & 0.34 & (1008) & & 1.29 & [1100N] & & $12 x$ & (אדונגו & & 123 & (1208) \\
\hline & \multirow{2}{*}{ oitus } & Sewon & $a \cdot x$ & 1.43 & 200 & 0.36 & 1.43 & 200 & 0.96 & 10 & 200 & 0.96 & 100 & 200 & 0.26 & 100 & 200 \\
\hline & & Year & & 1.48 & & & 1.44 & & & 100 & & & 140 & & & 140 & \\
\hline & \multirow{2}{*}{ Towal } & Sewon & 0.52 & 2.11 & 4.46 & 0.37 & 1.63 & c.OA & 0.97 & 200 & $s$ & 0.97 & 200 & ses & 0.37 & 200 & 3.15 \\
\hline & & Year & & 2.40 & & & 241 & (1018) & & $2 \pi$ & (1195) & & 274 & $(1230)$ & & 270 & (1119) \\
\hline
\end{tabular}


Table 10: Ray-tracing simulation - Combined daily average irradiation ( $\mathrm{kWh} \mathrm{m}^{-2}$ day ${ }^{-1}$ ) in Dublin

\begin{tabular}{|c|c|c|c|c|c|c|c|c|c|c|c|c|c|c|c|c|c|}
\hline & \multirow{2}{*}{\multicolumn{2}{|c|}{$\begin{array}{c}\text { Symmetrical } \\
\text { Tivted } 60^{\circ}\end{array}$}} & \multicolumn{3}{|c|}{ arr-TGo } & \multicolumn{3}{|c|}{ S-T6000 } & \multicolumn{3}{|c|}{ S.T60-100 } & \multicolumn{3}{|c|}{ S-T60-120 } & \multicolumn{3}{|c|}{ S-T60-140 } \\
\hline & & & wnrth & $\operatorname{mon} \alpha$ & suun & worm & $\operatorname{mon} \alpha$ & sumn & warth & soner & suMn & wimn & sonor & suma & wsm & $\operatorname{son} \alpha$ & suma \\
\hline \multirow{6}{*}{ 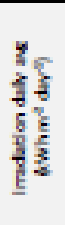 } & \multirow{2}{*}{ Direat } & Sansn & $1 \pi$ & תו & 453 & 0.37 & 530 & 7.51 & 100 & 5.16 & 7.32 & 2.10 & ABA & 6.4 & 102 & 4.51 & 3.58 \\
\hline & & Tess & & 245 & & & 47 & [มาร) & & 4.66 & [1968) & & 480 & (1220) & & 4.06 & (IIIS) \\
\hline & \multirow{2}{*}{ Diftas. } & Sansen & 0.90 & 0.98 & 100 & 0.90 & 0.98 & 100 & 0.90 & $0.8 s$ & 1.50 & 0.91 & 0.98 & 100 & 0.09 & 0.95 & 100 \\
\hline & & Tesr & & 0.96 & & & $0 . x$ & & & 0.96 & & & 0.96 & & & 0.96 & \\
\hline & \multirow{2}{*}{ Total } & Semsn & 210 & 400 & $6 \infty$ & 0.75 & 6.90 & 2.26 & 1.20 & 6.11 & 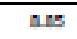 & $2.7 n$ & 5.00 & 7.57 & 200 & 5.47 & 7.11 \\
\hline & & Yesr & & $4 x$ & & & 50 & (1209) & & 5.61 & (12ns) & & 234 & (1220) & & 5.01 & [120) \\
\hline
\end{tabular}

\begin{tabular}{|c|c|c|c|c|c|c|c|c|c|c|c|c|c|c|c|c|c|}
\hline & \multirow{2}{*}{\multicolumn{2}{|c|}{$\begin{array}{c}\text { Symmetrical } \\
\text { Vertical }\end{array}$}} & \multirow{3}{*}{$\frac{\text { WNTh }}{2 \alpha x}$} & \multirow{3}{*}{$\begin{array}{l}\text { arr-y } \\
\text { moner } \\
2 \pi\end{array}$} & \multirow{3}{*}{$\frac{\text { sour }}{1 \text { CA }}$} & \multirow{3}{*}{$\frac{\text { WNTh }}{250}$} & \multicolumn{2}{|l|}{$=-v-\infty$} & \multicolumn{3}{|c|}{$5 v-100$} & \multicolumn{3}{|c|}{$3-v-1200$} & \multicolumn{3}{|c|}{ s-v-1600 } \\
\hline & & & & & & & $\operatorname{mon} \alpha$ & suMn & werth & $\operatorname{son} \alpha$ & SUMR & wsm & nonor & suma & wsth & nonoc & suma \\
\hline \multirow{6}{*}{ है } & \multirow{2}{*}{ Dirent } & Satusn & & & & & 0.00 & 0.12 & 290 & 0.90 & 0.12 & 250 & 100 & 0.12 & 2.41 & 2.12 & 0.18 \\
\hline & & Yess & & 2.29 & & & 0.97 & (1025) & & 1.17 & (515) & & 150 & $(\operatorname{ens})$ & & 2.91 & (7n) \\
\hline & \multirow{2}{*}{ Dithes. } & Satusn & 0.28 & $a r$ & 1.15 & 0.20 & $a r$ & 1.18 & 0.28 & $0 \pi$ & 1.15 & $0>0$ & $a r$ & 1.15 & $0>0$ & 0.72 & 1.18 \\
\hline & & Year & & $a r 2$ & & & $a r 2$ & & & 0.72 & & & ar & & & 0.72 & \\
\hline & \multirow{2}{*}{ Toan } & Sausn & 2.3 & 24 & $2 \pi$ & $2 m$ & 1.00 & 1.27 & $2 m 7$ & 1.71 & 1.27 & $2 m$ & 204 & 1.27 & 200 & 100 & 1.30 \\
\hline & & Yesr & & 100 & & & 100 & (96) & & 100 & [ess) & & 228 & (77) & & 252 & [125) \\
\hline
\end{tabular}

\begin{tabular}{|c|c|c|c|c|c|c|c|c|c|c|c|c|c|c|c|c|c|}
\hline & \multirow{2}{*}{\multicolumn{2}{|c|}{$\begin{array}{l}\text { Symmetrical } \\
\text { Horisontal }\end{array}$}} & \multicolumn{3}{|c|}{$\mathrm{BE}+1$} & \multicolumn{3}{|c|}{$=11 .-\infty$} & \multicolumn{3}{|c|}{ 5-11-100 } & \multicolumn{3}{|c|}{$5-11.1200$} & \multicolumn{3}{|c|}{$5-11-2000$} \\
\hline & & & warth & $\cos \alpha$ & suur & WNTn & $\operatorname{mon} \alpha$ & SUMR & WNTn & $\operatorname{son} \alpha$ & SUMn & wsth & Donor & suma & wimn & $\cos \alpha$ & suMg \\
\hline \multirow{6}{*}{ 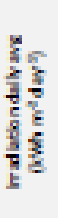 } & \multirow{2}{*}{ Direat } & Sansn & 0.07 & 208 & 4.16 & 0.58 & $a x$ & 5.67 & ase & 1.20 & S.at & 0.19 & $2 \pi$ & 6.00 & 0.50 & 2.91 & 500 \\
\hline & & Year & & 242 & & & 176 & (דימדן & & 2.02 & (MS) & & 206 & (12ח) & & 100 & (220) \\
\hline & \multirow{2}{*}{ Ditras. } & Stensen & 0.57 & 100 & 250 & 0.57 & 10 & 250 & 0.57 & 100 & 2.50 & 0.57 & 100 & 250 & 0.57 & 100 & 250 \\
\hline & & Yesr & & 149 & & & 205 & & & 1.43 & & & 149 & & & 203 & \\
\hline & \multirow{2}{*}{ Toral } & samsn & 120 & 103 & Gac & $a n$ & 200 & 7.97 & 0.02 & 200 & 7.98 & 0.70 & 4.19 & 1.90 & 1.07 & 4.94 & 7.50 \\
\hline & & Year & & 208 & & & 219 & (12) & & 2.45 & $(20 \times)$ & & 40 & (1910) & & 4.63 & (1128) \\
\hline
\end{tabular}

\begin{tabular}{|c|c|c|c|c|c|c|c|c|c|c|c|c|c|c|c|c|c|}
\hline & \multirow{2}{*}{\multicolumn{2}{|c|}{$\begin{array}{l}\text { Asymmetrical } \\
\text { Vertical }\end{array}$}} & \multicolumn{3}{|c|}{ arr-y } & \multicolumn{3}{|c|}{$n E-V-40$} & \multicolumn{3}{|c|}{ NeV. 50} & \multicolumn{3}{|c|}{ NEV-WE } & \multicolumn{3}{|c|}{ Ne-4.70 } \\
\hline & & & wNTh & $\operatorname{ton} \alpha$ & suur & WNTh & $\cos \alpha$ & sumn & WNTh & sonor & SUMR & wima & nonor & suma & with & Donor & suma \\
\hline \multirow{6}{*}{ 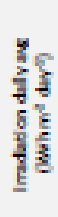 } & \multirow{2}{*}{ Direst } & Sewsn & $2 \alpha$ & $2 \pi$ & $10 \mathrm{~A}$ & 490 & 100 & 0.12 & 4.10 & 190 & 0.12 & 150 & 100 & 0.00 & 297 & 2.97 & תחם \\
\hline & & Yesr & & 2.29 & & & 200 & (1985) & & 2.85 & (1258) & & 292 & (1208) & & 207 & [125x) \\
\hline & \multirow{2}{*}{ Ditras } & swen & 0.20 & $0 \pi$ & 1.15 & 0.28 & $a \pi$ & 1.15 & 0.28 & $0 . \pi$ & 1.15 & $0 \geq 0$ & $a r$ & 1.15 & $0>0$ & 0.72 & 1.15 \\
\hline & & Yesr & & $a r$ & & & $a r$ & & & 0.72 & & & $a r$ & & & 0.72 & \\
\hline & \multirow{2}{*}{ Total } & Swasn & 2.21 & 24 & $2 \pi$ & Anto & 4.94 & 1.27 & 490 & 431 & 1.27 & 100 & 450 & 200 & 1.78 & 4.90 & 1.92 \\
\hline & & Year & & 200 & & & 201 & [127\%) & & 2.57 & [129s) & & 204 & (1218) & & 1.59 & (13\%) \\
\hline
\end{tabular}

\begin{tabular}{|c|c|c|c|c|c|c|c|c|c|c|c|c|c|c|c|c|c|}
\hline & Asym & tricol & & $\mathrm{BESH}$ & & & NG-11-40 & & & MS-11-Se & & & Ne+1-60 & & & $\mathrm{Ne+11.70}$ & \\
\hline & Hor & ntal & wom & $\cos \alpha$ & suur & WNTh & $\operatorname{mos} x$ & sumn & worn & nonor & sumn & wsm & soner & suma & wsm & moner & suma \\
\hline \multirow{6}{*}{ 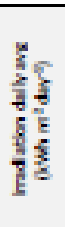 } & \multirow{2}{*}{ Dirst } & Semen & 0.07 & 20 & 4.16 & $a x$ & 206 & 2.4 & axe & 3.41 & 7.70 & 0.26 & 108 & 67 & 0.5 & 2. & 3.60 \\
\hline & & Tess & & 242 & & & 294 & [acss) & & 2.4 & (2518) & & 254 & (120) & & 1.25 & [בM: \\
\hline & \multirow{2}{*}{ Ditres. } & Stansn & 0.57 & 10 & 230 & 0.57 & 100 & 2.90 & 0.57 & 1.00 & 230 & 0.57 & 100 & 230 & 0.57 & 20 & 230 \\
\hline & & Yesr & & 240 & & & 140 & & & 1.49 & & & 240 & & & 201 & \\
\hline & \multirow{2}{*}{ Toen: } & Satens & 1.26 & $1 \mathrm{se}$ & G.ac & 0.02 & 500 & 1071 & 0.02 & $A B$ & 1000 & on & 490 & 2.00 & 0.92 & 4.91 & 7.50 \\
\hline & & Test & & 108 & & & 280 & (2600) & & 5.00 & [1925) & & 497 & (128) & & 4.69 & [121\$) \\
\hline
\end{tabular}

Table 12: Ray-tracing simulation - Combined daily average irradiation ( $k W h \mathrm{~m}^{-2}$ day $\left.^{-1}\right)$ in Ferrara

\section{RESULTS AND DISCUSSION}

The symmetrical CPCs mounted with an optimal fixed tilt show an increase of performance with each design in all locations. Overall all CPCs appear to have beneficial impact, however the smaller $\theta$ generate more concentration. The highest concentration ratios are achieved in Dublin, where they reach an overall increase of Idirect of nearly $200 \%$ annually. The simulation shows overall performance losses in every location when 
the symmetrical design is mounted vertically, for instance directly onto a façade. The estimated concentration ratios range between $26 \%$ and $88 \%$. The smaller $\theta$ are paired with the lowest concentration ratios. This poor performance can be attributed to CPC shading. The horizontal orientation of the symmetrical CPC is most beneficial in the southern location, closer to equator. This was to be expected as the maximum solar angle is highest in these locations. An overall increase between $119 \%$ for the wide $\theta$, and $137 \%$ for the narrow $\theta$ is estimated. The performance changes in Ferrara depending on $\theta$, and in Dublin, the simulated performance is poor (56\%-90\%). Similarly, this decrease in performance was to be expected due to lower maximum solar angles in locations at higher latitudes. The vertically mounted asymmetrical CPC achieved positive simulation results in most locations. This type appears to be more effective at higher altitudes, as the performance in Dublin was high (131\%). Interestingly, the highest concentration in these locations was achieved with intermediate $\theta$, indicating that here the optimal $\theta$ is near $50^{\circ}$ and $60^{\circ}$. In Ferrara a moderate concentration was achieved. Unsurprisingly, the vertically mounted asymmetrical CPCs perform best at high latitudes due to lower maximum sun angles. However, the performance is much better when compared to the vertically mounted symmetrical CPCs because the upper reflector is more open, allowing more exposure from above and creating far less solar shading. The horizontally mounted asymmetrical CPC has produced positive simulation results for most acceptance angles and locations. The optimal $\theta$ appears to be near $50^{\circ}$ in Dublin, with a concentration ratio of $137 \%$. In Ferrara the concentration ratio reaches up to $163 \%$ with $\theta=40^{\circ}$. Overall this type of CPC appears to generate good results everywhere, especially in Southern regions.

\section{CONCLUSION}

In this study, technical performance enhancement of BI CPC design types were assessed using ray-tracing simulations for two locations Dublin, Ireland and Ferrara, Italy to compare performance. A total of twenty-two 3-D models, each with unique symmetry, acceptance angles and orientations, were subjected to diffuse and direct solar ray-tracing simulations alongside reference models (without the application of CPC). The performance was assessed in each location during three pivotal days in the year (summer solstice; spring/autumn equinox; winter solstice). As a result, average hourly flux (Q) and daily irradiance (I) data was compiled for each CPC type. This information was used to determine annual performance estimations in energy production and economic benefits in terms of electricity savings and feed-in tariffs per locationCombining the results of the diffuse and direct solar ray-tracing simulations shows that the overall performance per $\mathrm{m}^{2} \mathrm{PV}$ surface can be enhanced using several CPC types. All tilted symmetrical CPCs increase annual absorber irradiance in each location (114\%-156\%) and horizontal asymmetrical CPCs are more effective at lower latitudes, whilst tilted symmetrical CPCs are more at higher latitudes. Asymmetrical CPCs have a higher performance than symmetrical CPCs when mounted strictly horizontally or vertically. In Dublin (IRL), CPC type S-T40-80 proved most effective with an estimated increase of annual irradiance from 2.64 to $4.11 \mathrm{kWh}^{-2}$ year $^{-1}$ (156\%). In Ferrara (ITA), CPC type S-T40-80 proved most effective once more with an estimated increase of annual irradiance from 4.38 to $5.67 \mathrm{kWh} \mathrm{m}^{-2}$ year $^{-1}$ (129\%). The simulation results clearly illustrated the potential effectiveness of application of some CPC types in various environments, providing increased solar irradiance of PV surfaces. The study presents detailed estimations of the technical performance of various CPC types in multiple locations. It provides a pragmatic overview of the estimated performances which can be used to inform decision-making regarding BIPV-CPC design and application.

\section{REFERENCES}

1. Tian, M., Su, Y., Zheng, H., Pei, G., Li, G. \& Riffat, S. 2018. A review on the recent research progress in (CPC) for solar energy applications. Renewable and Sustainable Energy Reviews, 82.

2. Jaaz, A. H., Hasan, H. A., Sopian, K., Ruslan, M. H. B. H. \& Zaidi, S. H. 2017. Design and development of compound parabolic concentrating for photovoltaic solar collector: Review. Renewable \& Sustainable Energy Reviews, 76, 11081121.

3. Zacharopoulos, A., Eames, P. C., McLarnon, D. \& Norton, B. 2000. Linear Dielectric Non-Imaging Concentrating Covers for PV Integrated Building Facades. Solar Energy, 68, 439-452. 
4. Mallick, T. K., Eames, P. C., Hyde, T. J. \& Norton, B. 2004. Design and experimental characterisation of an asymmetric CPC PV for building façade integration in the UK. Solar Energy, 77, 319-327.

5. Mallick, T. K., \& Eames, P. C., 2007. Design and fabrication of low concentrating second generation PRIDE concentrator. Solar Energy Materials and Solar Cells, 91, 597-608.

6. Baig, H., Sarmah, N., Chemisana, D., Rosell, J. \& Mallick, T. K. 2014. Enhancing performance of a linear dielectric based concentrating PV system. Energy, 73, 177-191.

7. Sarmah, N., Richards, B. S. \& Mallick, T. K. 2014. Design, development and indoor performance analysis of a low concentrating dielectric PV module. Solar Energy, 103, 390-401.

8. Ortega A. Design and characterization of a roof-mounted compound parabolic concentrator with luminescent down sifting layers. MSc Thesis. Trinity College Dublin, Dublin, Ireland. 2017. 